\title{
The Role of PET/CT to Detect Bone Marrow Involvement in Hodgkin Lymphoma
}

(i) Ali ESER, io Funda PEPEDIL TANRIKULU, id Aslıhan SEZGiN, id Ayşe Tülin TUĞLULAR

Marmara University Faculty of Medicine, Department of Internal Diseases, İstanbul, Turkey

\begin{abstract}
Objective: Bone marrow involvement (BMI) is a significant component of staging of Hodgkin's lymphoma (HL). Unilateral bone marrow biopsy (BMB) from dorsal iliac bone is the standard method to determine BMI. Positron emission tomography/computerized tomography (PET/CT) is recommended as a complimentary technique to determine BMI and to evaluate response to treatment. The aim of this study is to determine whether PET/CT can replace BMB to detect BMI in patients with HL.

Methods: A total of 159 patients diagnosed as having HL were evaluated retrospectively. One hundred and four patients who met the criteria were included in the study. BMB and PET/CT were performed on all patients during initial staging.

Results: Of the 104 patients, 44 (42.3\%) and 17 (16.3\%) had BMI in PET/CT and BMB respectively. All patients who had BMI in BMB also had involvement in PET/CT. BMB did not detect BMI in 27 patients who had BMI in PET/CT. All 27 patients had partial or complete remission on PET/CT performed at the end of treatment. This finding was regarded as a relative indicator for BMI. PET/CT had a sensitivity and negative predictive value of $100 \%$, specificity of $68.9 \%$ and accuracy rate of $74 \%$ to determine BMI.

Conclusion: We observed that PET/CT and BMB were compatible to determine BMI in patients diagnosed with HL. Unilateral BMB may result false negative especially in cases with focal involvement. Therefore, PET/CT should be considered as a complimentary technique to determine BMI. Staging should be reevaluated in patients who have BMI in PET/CT and a negative BMB treatment should be planned accordingly.
\end{abstract}

Keywords: Hodgkin's lymphoma, positron emission tomography, bone marrow biopsy, bone marrow involvement

\section{Introduction}

Hodgkin's lymphoma (HL) comprises $12 \%$ of all lymphomas and $1 \%$ of all malignancies (1). It is possible to achieve cure with modern first line treatment modalities in about $90 \%$ of patients. However treatment fails in 10\% of early stage and 30\% of advanced stage disease. Cure rates are around $50 \%$ in relapsed disease, in extra-nodal disease and in patients relapsing early after treatment of advanced stage disease (2-5). Correct staging is necessary for an effective treatment plan $(6,7)$. Bone marrow involvement (BMI) is one of the most important prognostic factors in lymphomas. BMI corresponds to stage IV disease according to the Ann Arbor staging system (8). Staging of HL is based on the Cotswold's modification of the Ann-Arbor system $(8,9)$ and commonly includes computerized tomography (CT) and bone marrow biopsy (9). BMI is detected in $5-14 \%$ of patients with $\mathrm{HL}$ $(10,11)$. Unilateral bone marrow biopsy (BMB) performed from dorsal iliac crest is considered as standard and performed routinely during staging $(12,13)$. However main disadvantages of BMB are its interventional nature and it allows only a limited region of bone marrow to be investigated. A previous study reported $80 \%$ false negativity of a unilateral iliac crest biopsy compared with a bilateral biopsy in patients with lymphoma (14). Imaging

Address for Correspondence: Funda PEPEDiL TANRIKULU, Marmara University Faculty of Medicine, Department of İnternal Diseases, İstanbul, Turkey 
techniques may be used to detect bone marrow involvement, CT may be able to show bone lesions or late occurring bone changes; however its sensitivity in early stage disease is low (7). Magnetic resonance imaging (MRI) is a sensitive technique although it is not used widely in routine practice. F-18 fluorodeoxyglucose (FDG) positron emission tomography/computerized (PET/CT) has been used for staging and evaluating response in patients with lymphoma. A number of studies have shown that PET/CT is useful to evaluate bone marrow $(6,7,15)$. It has been shown that PET/CT and BMB are $78-82 \%$ compatible, thus PET/CT is recommended as a complimentary technique (14-17).

The aim of this study is to compare BMB and FDG-PET/CT to detect BMI and to determine whether FDG-PET/CT can replace $\mathrm{BMB}$ in patients with HL.

\section{Methods}

Patients over 18 years old who had been diagnosed as having HL between January 2007 and January 2015 in our University Hospital were evaluated retrospectively. Patients who did not have bone marrow biopsy and PET/CT performed simultaneously at diagnosis were excluded from the study. All patients had BMB and PET/CT performed at diagnosis. Age, sex, date of diagnosis, type of PET/CT involvement (focal, diffuse), $\mathrm{SUV}_{\text {max- values }}$, BMI, stage of disease, risk score, B symptoms, date of last follow up, treatments, and PET/CT results before and after treatment were recorded.

Administration of PET/CT was carried out locally on dedicated machines in accordance with at the manufacturer guidelines Marmara University Pendik Training and Research Hospital. During the evaluation of PET/CT, bone marrow activity greater than that of liver was considered positive for BMI by HL unless definitively explained by an alternate cause. Involvement was evaluated as diffuse or focal (1,2,3 and $>3$ areas).

All bone marrow samples were obtained unilaterally from dorsal iliac crest and each was $15-20 \mathrm{~mm}$ in length. Samples were evaluated by an expert hematopathologist.

Since our study was conducted retrospectively, it was not feasible to get informed consent from the patients, however it was approved by the Instutional ethical review board. Marmara University Faculty of Medicine Clinical Research Ethics Committee, Approval no: 09.2013.0242.

\section{Statistical Analysis}

BMB was considered as standard reference to determine BMI. Results that were compatible with BMB and PET/CT were regarded as true positive and true negative initially. Cases that were PET/CT positive and BMB negative initially were evaluated with PET/CT during and at the end of treatment. Sensitivity, specificity, negative and positive predictive values were calculated. PASW 18.0 for Windows software was used for the statistical analysis. Descriptive statistics were expressed as average, standard deviation, median, percentile 25 (Q1), percentile 75 (Q3), minimum and maximum. Conformity of the variables to the normal distribution was analyzed with visual (histogram and probability diagram) and analytical (Kolmogorov-Simirnov/ Shapiro-Wilktests) methods. The paired group comparison was conducted by the Mann-Whitney $U$ test for the diagnosis age, $\mathrm{SUV}_{\max }$, tracking duration and phase, the IPS and PET phase escalation which were not normally distributed. The chi-square test was used for the paired comparison between the categorical variable, and the Fisher exact test was used where the chi-square condition was unable to be provided. Cases where type-1 error was below $5 \%$ were regarded as statistically significant.

\section{Results}

A total of 159 patients who had been diagnosed as having HL between January 2007 and January 2015 in Marmara University Medical Faculty were evaluated retrospectively. Fifty-five patients who did not have bone marrow biopsy and PET/CT performed simultaneously at diagnosis were excluded from the study. A total of 104 patients were evaluated.

Both PET/CT and BMB were performed for initial staging in a total of 104 newly diagnosed patients with HL ( 45 females and 59 males). Median age was 32 (18-80) years and $94.2 \%$ of the patients were diagnosed as having classical HL $(58.3 \%$ nodular sclerosis, $28.2 \%$ mixed cellularity, $5.8 \%$ lymphocyte rich, $1.9 \%$ lymphocyte depleted), and the remaining $5.8 \%$ was diagnosed as having nodular lymphocyte predominant type. One patient had stage I, 36 patients had stage II, 37 patients had stage III and 30 patients had stage IV disease. According to IPS risk score; 67 patients had low, 17 intermediate and 18 high risk. Clinical characteristics of patients are summarized in Table 1.

BMI was detected in 44 patients (42.3\%) with PET/CT and in 17 patients (16.3\%) with BMB. Out of 44 patients who had BMI in PET/CT, 27 did not have BMI in BMB, however all 17 patients who had BMI in BMB also had involvement in PET/ CT (Table 2). Two patients with stage II and 17 patients with stage III disease were regarded as stage IV after restaging with PET/CT

Disease stage and higher IPS risk were statistically significant in patients who were PET positive for BMI compared to PET negative patients $(\mathrm{p}<0.001$ and $\mathrm{p}<0.001$, respectively).

Disease stage and higher IPS risk were statistically significant in patients who had BMI in bone marrow biopsy compared to patients who were bone marrow biopsy negative $(\mathrm{p}<0.001$ and $\mathrm{p}<0.001$, respectively).

PET/CT was negative for BMI in 60 patients. All of these patients were also negative for BMI in BMB. Remaining 44 patients were $\mathrm{PET} / \mathrm{CT}$ positive. There were not any uncertain cases since FDG values greater than liver was considered as positive. Involvement was evaluated as focal or diffuse. Focal group was classified as $1,2,3$, and $>3$ areas. According to this classification 3 patients had focal 1, 4 patients had focal 2, 6 patients had focal 3 and 
1 patient had focal $>3$ areas of involvement with a total of 28 patients having focal involvement. Sixteen patients had diffuse involvement. PET/CT positive, BMB negative patients had a median $S U_{\text {max-value }}$ of 5.1 while $\mathrm{PET} / \mathrm{CT}$ positive, $\mathrm{BMB}$ positive patients had 6.8 which was not statistically significant.

Table 1. Demographic features of patients

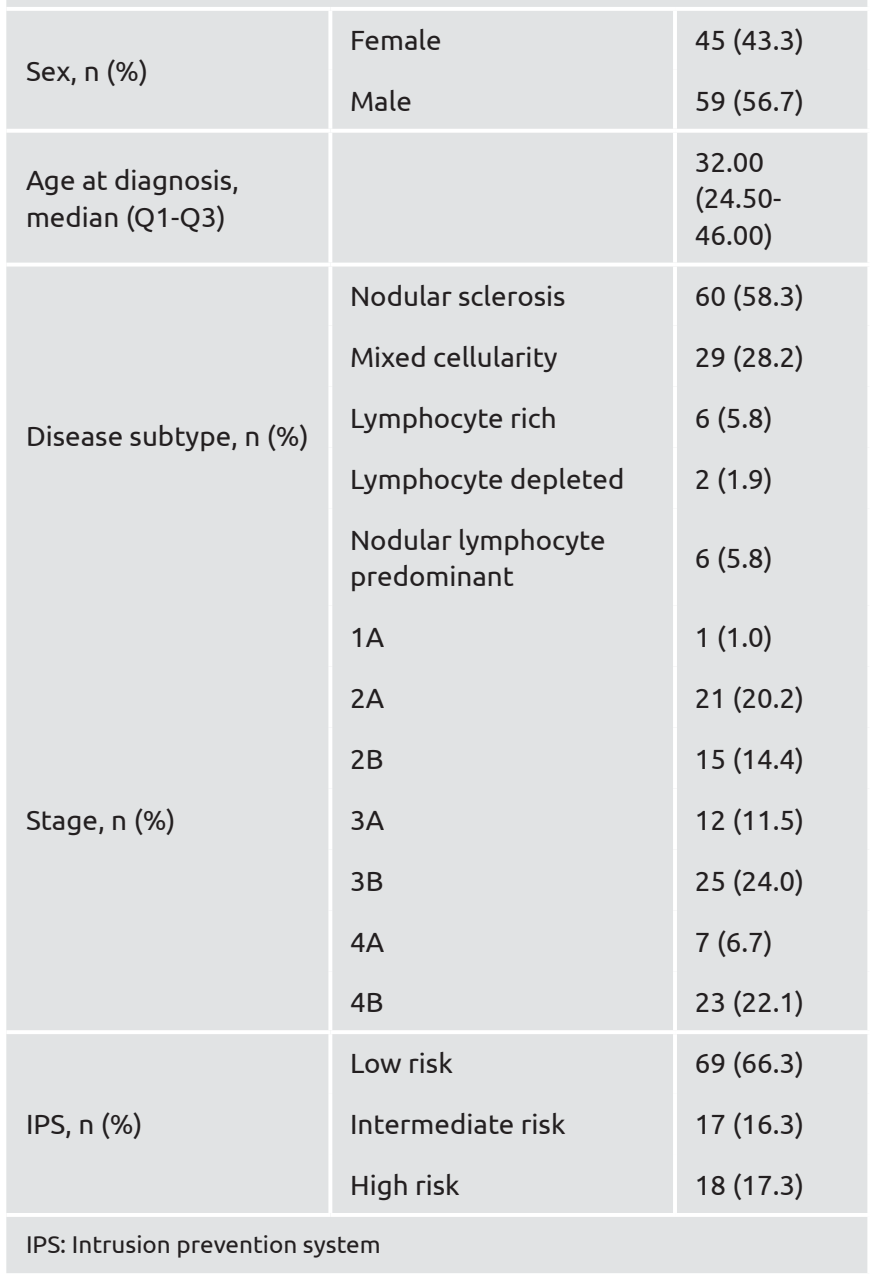

Table 2. Bone marrow involvement

\begin{tabular}{|c|c|c|}
\hline & & $n(\%)$ \\
\hline $\begin{array}{l}\text { Bone marrow involvement } \\
\text { in PET/CT }\end{array}$ & & $\begin{array}{l}44 \\
(42.3)\end{array}$ \\
\hline $\begin{array}{l}\text { Bone marrow involvement } \\
\text { in bone marrow biopsy }\end{array}$ & & $\begin{array}{l}17 \\
(16.3)\end{array}$ \\
\hline \multirow{2}{*}{$\begin{array}{l}\text { PET/CT -Bone marrow } \\
\text { biopsy }\end{array}$} & $\begin{array}{l}\text { PET/CT (+) } \\
\text { Bone marrow biopsy } \\
(-)\end{array}$ & $\begin{array}{l}27 \\
(61.4)\end{array}$ \\
\hline & $\begin{array}{l}\text { PET/CT (+) } \\
\text { Bone marrow biopsy } \\
(+)\end{array}$ & $\begin{array}{l}17 \\
(38.6)\end{array}$ \\
\hline
\end{tabular}

When evaluating involvement type in PET/CT, it was observed that as the areas of focal involvement increased, the rate of involvement in $\mathrm{BMB}$ also increased. $\mathrm{BMB}$ was positive in all cases with diffuse involvement. Increase in disease stage in patients who had BMI in PET/CT was statistically significantly higher thanpatients who were negative for $(\mathrm{p}<0.001)$. All patients who had BMI in PET/CT at diagnosis had partial or complete regression after treatment. Twenty-two (81.5\%) of PET/CT positive cases had complete regression and $5(18.5 \%)$ had partial regression, a finding that was regarded as a sign of BMI (Table 3).

Analysis revealed a sensitivity of $100 \%$ for PET/CT to detect BMI, while specificity was found to be $68.97 \%$ (Table 4).

Table 3. Comparison of bone marrow biopsy positive and negative groups within the positron emission tomography/ computerized tomography positive patients

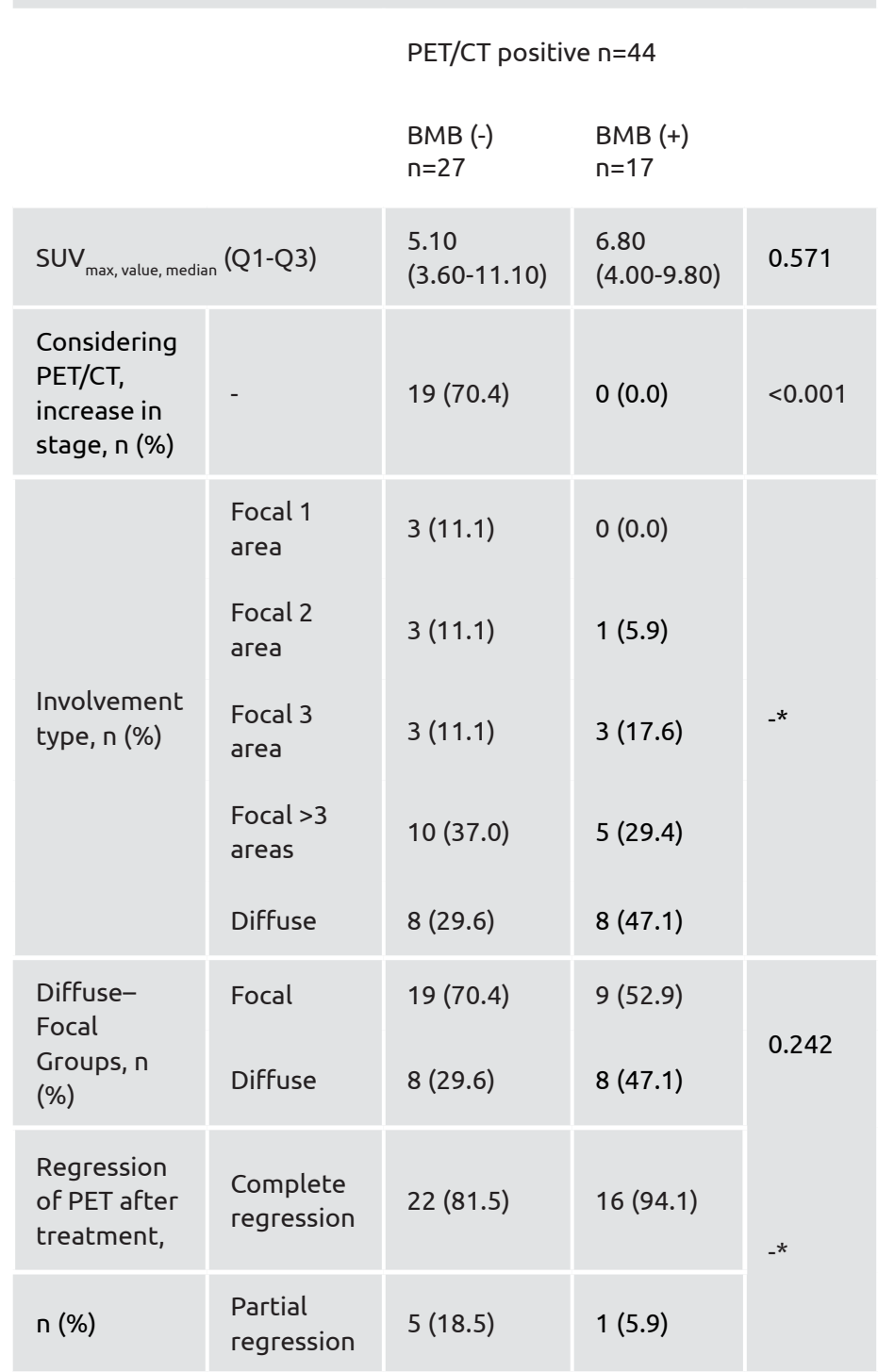

*Analysis was not performed due to low patient numbers, PET/CT: Positron emission tomography, computerized tomography 
Table 4. Positron emission tomography-bone marrow biopsy sensitivity, specificity and positive-negative predictive values

\begin{tabular}{|c|c|c|c|}
\hline & \multirow{2}{*}{ Value } & \multicolumn{2}{|c|}{ Confidence Interval } \\
\hline & & Lower Limit & Upper Limit \\
\hline Sensitivity & $100.00 \%$ & $80.49 \%$ & $100.00 \%$ \\
\hline Spesificity & $68.97 \%$ & $58.14 \%$ & $78.45 \%$ \\
\hline $\begin{array}{l}\text { Positive } \\
\text { predictive value }\end{array}$ & $38.64 \%$ & $24.36 \%$ & $54.50 \%$ \\
\hline $\begin{array}{l}\text { Negative } \\
\text { predictive value }\end{array}$ & $100.00 \%$ & $94.04 \%$ & $100.00 \%$ \\
\hline Accuracy rate & $74.04 \%$ & $64.52 \%$ & $82.14 \%$ \\
\hline
\end{tabular}

\section{Discussion}

Anatomical extensiveness and to a lesser degree histological subtype of the disease are factors that define prognosis and guide treatment in HL. Aim of treatment is to achieve cure with minimal complications. Therefore, correct staging is necessary to make an effective treatment plan. Evaluating BMI is an important step in staging. BMI is detected in about $10 \%$ of patients with HL (10). In our study, BMI was detected in $16.3 \%$ of patients, which was higher than previously reported. Standard procedure to evaluate BMI is to perform unilateral BMB. BMI may be focal or diffuse. Blind biopsy samples may reveal false negative results if performed from uninvolved areas of bone marrow and BMI should be checked with other techniques. Positivity of bilateral biopsy is $10-50 \%$ which is higher than unilateral biopsy. Therefore, standard $\mathrm{BMB}$ does not reflect a correct evaluation of bone marrow (18). A negative BMB does not rule out BMI if imaging techniques show abnormalities. BMI is nodular in $65 \%$ of patients with HL on MRI. This explains a negative BMB when BMI is in other areas (19). It has been suggested that PET/ CT could replace blind BMB or could be used as a guide to find the correct area for BMB. For patients with a high risk of BMI, FDG may be decisive. Since PET/CT detects a greater area than $\mathrm{BMB}$, if there is not any FDG uptake, then there is no BMI (15). Therefore it is more sensitive than $\mathrm{CT}$ and bone scintigraphy to detect BMI. PET/CT may reveal early BMI in patients with a negative CT scan $(6,7,10)$.

For this reason, true positivity should be determined. PET/CT results are evaluated in 3 reference levels in PET/CT positive cases. First of all, if BMB is positive, this is a verification at the highest level. Secondly, skeletal PET/CT findings of multifocal pattern (3 or more skeletal lesions): This pattern is typical for $\mathrm{HL}$ and may rarely be seen in other diseases (6). Thirdly, complete or near complete regression in PET/CT after chemotherapy is an intermediate level of verification, since FDG uptake may be observed in inflammatory and traumatic lesions as well. In our study, twenty-seven patients who had BMI in PET/CT but not in $\mathrm{BMB}$ were re-evaluated after treatment. Partial or complete regression of BMI was regarded as a clue for true positivity.

PET/CT should not be regarded as positive when FDG uptake is diffuse, homogeneous and symmetrical in axial and appendicular skeleton unless the FDG uptake is overt. It is regarded as bone marrow activation when FDG uptake of bone marrow is greater than liver. PET/CT may result false positive when there is a concomitant systemic disease or granulocyte colony stimulating factor use. Such cases and benign increases due to inflammatory reactions may be interpreted as false positivity, however BMI cannot be ruled out $(7,20)$.

Most studies include a heterogeneous population of Non Hodgkin's and HL. This results in a variety of FDG uptake due to different subtypes (18). In our research, solely patients with HL were evaluated.

In a study conducted by Cetin et al. (21), 61 patients with HL were analyzed. Although, concordant results were revealed between PET/CT and BMB in 52, there were 2 patients with positive $\mathrm{BMB}$ while $\mathrm{PET} / \mathrm{CT}$ was negative. On the other hand, 7 patients had negative BMB, while they were positive on PET/CT (21). In contrast, our study did not reveal any false negative cases.

Moulin-Romsee et al. (7). reported 83 patients diagnosed as having HL. Seven patients had BMI with BMB. Eighteen patients had BMI with PET/CT. Out of 11 patients that were PET/CT positive and BMB negative, 2 had stage IV disease due to lung and liver involvement. Remaining 9 patients were considered as having disseminated disease and treated accordingly (22). In our study, 2 patients with stage II and 17 patients with stage III disease initially were regarded as having stage IV disease after detecting BMI with PET/CT and treatment plans were reevaluated.

We showed that sensitivity and negative predictive value of PET/ CT were $100 \%$. Specificity was $68.9 \%$ and accuracy rate was 74 $\%$. These findings were similar to the data published by Pakos et al. (18). In a study by Anjum Bashir Khan et al. (23), 130 patients diagnosed as having lymphoma were evaluated. PET/CT and BMB proved BMI in $35(27 \%)$ patients. PET/CT detected BMI in $33(94 \%)$ of these patients, while BMB detected in 14 (40\%) patients (23). Sensitivity and specificity of PET/CT to detect BMI was $94 \%$ and $100 \%$, respectively. Sensitivity of BMB was found to be $40 \%$ and specificity was $100 \%$. Accuracy rate was $98.5 \%$ for PET/CT and $84 \%$ for BMB. We observed similar results for sensitivity and specificity in our current study.

Although, our study confirmed studies previously performed, the most important limitation of our study was the relative small number of study population and its retrospective nature. Therefore, prospective studies including larger patient populations are necessary.

\section{Conclusion}

FDG-PET/CT increased the sensitivity of detecting BMI compared to conventional staging. In patients who have BMI 
according to PET/CT but not according to BMB, staging should be re-evaluated and treatment should be planned accordingly. Absence of false positive results in PET/CT and the observation of regression in FDG uptake after treatment in all patients may be an indicator to show PET/CT could replace BMB to determine BMI.

\section{Ethics}

Ethics Committee Approval: Marmara University Faculty of Medicine Clinical Research Ethics Committee, Approval no: 09.2013 .0242 .

Informed Consent: Since our study was conducted retrospectively, it was not feasible to get informed consent from the patients, however it was approved by the instutional ethical review board.

Peer Review: Externally peer-reviewed.

\section{Authorship Contributions}

Concept: A.E., F.P.T., Design: A.E., A.T.T., Data Collection or Processing: A.E., Analysis or Interpretation: A.E., F.P.T., Literature Search: A.E., A.S., Writing: A.E., A.S.

Conflict of Interest: No conflict of interest was declared by the authors.

Financial Disclosure: The authors declared that this study received no financial support.

\section{References}

1. Diehl V. Hodgkin's disease-from pathology specimen to cure. N Engl J Med 2007;357:1968-71.

2. Brice P, Bastion Y, Divine M, Nedellec G, Ferrant A, Gabarre J, et al. Analysis of prognostic factors after the first relapse of Hodgkin's disease in 187 patients. Cancer 1996;78:1293-9.

3. Moskowitz $\mathrm{CH}$, Nimer SD, Zelenetz AD, Trippett T, Hedrick EE, Filippa DA, et al. A 2-step comprehensive high-dose chemoradiotherapy second-line program for relapsed and refractory Hodgkin disease: analysis by intent to treat and development of a prognostic model. Blood 2001;97:616-23.

4. Josting A, Franklin J, May M, Koch P, Beykirch MK, Heinz J, et al. New prognostic score based on treatment outcome of patients with relapsed Hodgkin's lymphoma registered in the database of the German Hodgkin's lymphoma study group. J Clin Oncol 2002;20:221-30.

5. Brice P. Managing relapsed and refractory Hodgkin lymphoma. Br J Haematol 2008;141:3-13.

6. Pelosi E, Penna D, Deandreis D, Chiappella A, Skanieti A, Vitolo U, et al. FDG-PET in the detection of bone marrow disease in Hodgkin's disease and aggressive non-Hodgkin's lymphoma and its impact on clinical management. Q J Nucl Med Mol Imaging 2008;52:9-16.

7. Moulin-Romsee G, Hindie $\nabla \mathrm{E}$, Cuenca X, Brice P, Decaudin D, Benamor M, et al. 18F-FDG PET/CT bone/bone marrow findings in Hodgkin's lymphoma may circumvent the use of bone marrow trephine biopsy at diagnosis staging. Eur J Nucl Med Mol Imaging 2010;37:1095-105.

8. Carbone PP, Kaplan HS, Musshoff K, Smithers DW, Tubiana M. Report of the Committee on Hodgkin's Disease Staging Classification. Cancer Res 1971;31:1860-1.

9. Lister TA, Crowther D, Sutcliffe SB, Glatstein E, Canellos GP, Young RC, et al. Report of a committee convened to discuss the evaluation and staging of patients with Hodgkin's disease: Cotswolds meeting. J ClinOncol 1989;7(11):1630-6. Erratum in: J Clin Oncol 1990;8:1602.

10. Levis A, Pietrasanta D, Godio L, Vitolo U, Ciravegna G, Di Vito F, et al. A large-scale study of bone marrow in- volvement in patients with Hodgkin's lymphoma. Clin Lymphoma 2004;5:50-5.

11. Ponzoni M, Ciceri F, Crocchiolo R, Famoso G, Doglioni C. Isolated bone marrow occurrence of classic Hodgkin's lymphoma in an HIVnegative patient. Haematologica 2006;91:ECR04.

12. Brusamolino E, Bacigalupo A, Barosi G, Biti G, Gobbi PG, Levis A, et al. Classical Hodgkin's lymphoma in adults: guidelines of the Italian Society of Hematology, the Italian Society of Experimental Hematology, and the Italian Group for Bone Marrow Transplan- tation on initial work-up, management, and follow-up. Haematologica 2009;94:550-65.

13. Howell SJ, Grey M, Chang J, Morgenstern GR, Cowan RA, Deakin $\mathrm{DP}$, et al. The value of bone marrow examination in the staging of Hodgkin's lymphoma: a review of 955 cases seen in a regional cancer centre. Br J Haematol 2002;119:408-11.

14. Wang J, Weiss LM, Chang KL, Slovak ML, Gaal K, Forman SJ, et al. Diagnostic utility of bilateral bone marrow examination: significance of morphologic and ancillary technique study in malignancy. Cancer 2002;94:1522-31.

15. Moog F, Bangerter M, Kotzerke J, Guhlmann A, Frickhofen N, Reske SN. 18-F-fluorodeoxyglucose-positron emission tomography as a new approach to detect lymphomatous bone marrow. J Clin Oncol 1998;16:603-9.

16. Hong J, Lee Y, Park Y, Kim SG, Park SH, Jeong J, et al. Role of FDGPET/CT in detecting lymphomatous bone marrow involvement in patients with newly diagnosed diffuse large B-cell lymphoma. Ann Hematol 2012;91:687-95.

17. Fuster D, Chiang S, Andreadis C, Guan L, Zhuang H, Schuster S, et al. Can [18F]Fluorodeoxyglucose positron emission tomography imaging complement biopsy results from the iliac crestfor the detection of bone marrow involvement in patients with malignant lymphoma? Nucl Med Commun 2006;27:11-5.

18. Pakos EE, Fotopoulos AD, Ioannidis JP. 18F-FDG PET for evaluation of bone marrow infiltration in staging of lymphoma: A meta-analysis. J Nucl Med 2005;46:958-63.

19. Inoue K, Goto R, Okada K, Kinomura S, Fukuda H. A bone marrow 18F-FDG uptake exceeding the liver uptake may indicate bone marrow hyperactivity. Ann Nucl Med 2009;23:643-9.

20. Agool A, Glaudemans AW, Boersma HH, Dierckx RA, Vellenga E, Slart RH. Radionuclide imaging of bone marrow disorders. Eur J Nucl Med Mol Imaging 2011;38:166-78.

21. Cetin G, Cıkrıkcıoglu MA, Ozkan T, Karatoprak C, Ar MC, Eskazan $\mathrm{AE}$, et al. Can Positron Emission Tomography and Computed 
Tomography be a substitude for bone marrow biopsy in detection of bone marrow involvement in patients with Hodgkin's lymphoma and Non-hodgkin's lymphoma. Turk J Hematol 2015;32:213-9.

22. Moulin-Romsee G, Hindie E, Cuenca X, Brice P, Decaudin D, Benamor $\mathrm{M}$, et al. FDG PET/CT bone/bone marrow findings in Hodgkin's lymphoma may circumvent the use of bone marrow trephine biopsy at diagnosis staging Eur J Nucl Med Mol Imaging 201037:1095-105.

23. Khan AB; Barrington SF, Mikhaeel NG, Hunt AA, Cameron L, Morris T, et al. PET-CT Staging of DLBCL accurately identifies and provides new insight into the clinical significance of bone marrow involvement. Blood 2013;122:61-7. 